\title{
Atributos do Solo Relacionados com a Produção da Castanheira-do-Brasil (Bertholletia excelsa)
}

\author{
Mirian Gomes Costa ${ }^{1}$, Helio Tonini² ${ }^{2}$ Paulo Mendes Filho ${ }^{1}$ \\ ${ }^{1}$ Departamento de Ciências do Solo, Universidade Federal do Ceará - UFC, Fortaleza/CE, Brasil \\ ${ }^{2}$ Empresa Brasileira de Pesquisa Agropecuária - Embrapa Agrossilvipastoril, Sinop/MT, Brasil
}

\begin{abstract}
RESUMO
Neste estudo foram analisados os atributos do solo relacionados com variações na produção de frutos da castanheira bem como o estado nutricional da espécie. As árvores foram selecionadas em parcela permanente (Caracaraí, RR) e divididas em quatro classes de produção: alta, média, baixa e muito baixa. Em novembro de 2009, amostras de solo e folhas foram coletadas para se avaliar a fertilidade do solo, o número de esporos de fungos micorrízicos arbusculares e o estado nutricional das árvores. Árvores com maior produção de frutos ocorreram associadas a maior teor de $\mathrm{Al}$ trocável na camada de $0-20 \mathrm{~cm}$ do solo, bem como a menor valor de $\mathrm{pH} \mathrm{e}$ saturação por bases. A variável discriminante resultante deste estudo não foi considerada como responsável por interferir no potencial produtivo da castanheira-do-brasil, mas serviu como indicativo de que árvores mais produtivas contribuem na remoção de bases trocáveis, favorecendo a acidificação do solo.
\end{abstract}

Palavras-chave: extrativismo, acidez do solo, absorção de bases, Roraima.

\section{Soil Attributes Related with Production of Brazil Nut Tree (Bertholletia excelsa)}

\begin{abstract}
In this study we analyzed the soil attributes related with variation in the fruit production of Brazil nut trees, as well as the nutritional status of the species. Trees were selected in permanent plot (Caracaraí - RR) and divided in four production classes: High, Medium, Low and Very low. In November 2009, soil and leaves samples were taken to evaluate soil fertility, soil colonization by arbuscular mycorrhizal fungi, and trees nutritional status. Trees with higher fruits production occurred associated with higher exchangeable $\mathrm{Al}$ in soil depth of $0-20 \mathrm{~cm}$, as well as to smaller $\mathrm{pH}$ and bases saturation values. The discriminant variable resulting of the present study was not assumed as responsible for interfering with the productive potential of Brazil nut trees; however, it indicates most productive trees contributing with exchangeable basis removal, favoring soil acidification.
\end{abstract}

Keywords: extractivism, soil acidity, bases uptake, Roraima.

\section{INTRODUÇÃO E OBJETIVOS}

A castanheira-do-brasil (Bertholletia excelsa Humb. e Bonpl., Lecythidaceae) é uma espécie encontrada em estado nativo na Amazônia. A comercialização das amêndoas representa atividade econômica basicamente extrativista. Devido aos altos teores de selênio encontrados nas amêndoas (Kluczkovski et al., 2015), 
a castanha-do-brasil, como alimento, contribui na prevenção de doenças (Rayman, 2002; Huguenin et al., 2015) e o seu reconhecimento como alimento funcional aumenta o apelo para o seu consumo.

A coleta da castanha-do-brasil caracteriza uma forma de exploração que pode ser estratégica na conservação das florestas, pois a partir do momento em que há geração de renda nas comunidades com esse tipo de exploração, deve haver contenção de práticas não conservacionistas de uso do solo (Ticktin, 2004; Wunder et al., 2014). Adicionalmente, a introdução da castanheira-do-brasil em sistemas agroflorestais (SAF) pode tornar sustentável o uso do solo em áreas alteradas na Amazônia, com a exploração não só de árvores em populações naturais, mas também em florestas plantadas.

Entretanto, para o manejo da espécie é importante conhecer suas características e exigências para plena produção. Tonini et al. (2008) observaram baixa produção de frutos e sementes em castanhais de Roraima em comparação com castanhais existentes em outras localidades da Amazônia. Atributos relacionados ao solo e à disponibilidade de nutrientes foram citados como fatores importantes para a produção de frutos de castanheiras (Viana et al., 1998; Zuidema, 2003). Camargos (1999) encontrou sintomas visuais de deficiência para todos os nutrientes na castanheira, com exceção do fósforo $(\mathrm{P})$, cobre $(\mathrm{Cu})$ e zinco $(\mathrm{Zn})$. Schroth et al. (2015) constataram que a castanheira-do-brasil responde à aplicação de calcário e de doses de fertilizantes, com melhorias no crescimento e no teor foliar de nutrientes.

Kainer et al. (2007) constataram que a capacidade de troca cationnica (CTC) e o P extraível do solo explicaram variações na produção de castanheiras. Segundo os autores, a CTC apresentou correlação positiva, enquanto que o teor de $\mathrm{P}$ extraível apresentou fraca correlação negativa com a produção de frutos. Ainda no estudo citado, a CTC do solo no castanhal avaliado foi maior em relação aos valores normalmente encontrados em outros Argissolos da Amazônia (Lathwell \& Grove, 1986).

Com relação ao $\mathrm{P}$, uma possível explicação para a fraca correlação negativa entre o teor extraível do elemento no solo e a produção de frutos da castanheira-do-brasil seria a interação simbiótica das árvores com fungos micorrízicos arbusculares. Os fungos micorrízicos produzem extensa rede de micélios e essa pode constituir mais de $25 \%$ do total da biomassa microbiana do solo (Olsson et al., 1999). Os micélios mobilizam P e outros nutrientes essenciais, melhorando a nutrição da planta (Bernatchez et al., 2008).

Assume-se como hipótese que o teor foliar de $\mathrm{P}$ e a produtividade das castanheiras não estão relacionados com os baixos teores de $\mathrm{P}$ no solo, pois a presença de fungos micorrízicos arbusculares na rizosfera das plantas garante o suprimento do nutriente para a espécie. Supõe-se também que deve haver outro atributo químico do solo que melhor explique a baixa produtividade das árvores. Este estudo foi conduzido com o objetivo de analisar atributos químicos do solo, a colonização do solo por fungos micorrízicos arbusculares, bem como de determinar o estado nutricional de castanheiras com diferentes níveis de produção de frutos.

\section{MATERIAL E MÉTODOS}

\section{1. Área de estudo e árvores avaliadas}

As árvores foram selecionadas em parcela permanente $(300 \times 300$ metros $)$ instalada em castanhal em Caracaraí, RR, nas coordenadas $1^{\circ} 48^{\prime} 58^{\prime \prime}$ de latitude norte e $61^{\circ} 7^{\prime} 41^{\prime \prime}$ de longitude oeste. A área apresenta relevo plano a ondulado com vegetação predominante de floresta tropical úmida (Brasil, 1975). O clima na região é classificado como Ami, com precipitação média anual entre 1.700-2.000 mm. O período chuvoso ocorre de abril a agosto, com totais mensais superiores a $100 \mathrm{~mm}$. A partir de setembro há redução nas chuvas, com período seco ocorrendo de novembro a março. A temperatura média anual é de $27^{\circ} \mathrm{C}$ (FEMACT, 1993).

Foram selecionados 19 indivíduos adultos (DAP > $50 \mathrm{~cm}$ ) dominantes, com boa forma de copa e livres de infestação por cipós. Os indivíduos abrangeram a variação existente na produção das castanheiras, representada pelo número de frutos por árvore, observada em monitoramento anual realizado de 2006 a 2009. As árvores foram divididas em quatro classes de produção: A - alta (340 a 566 frutos); B - média (226 a 339 frutos); C - baixa (113 a 225 frutos); e D - muito baixa (0-112 frutos). Foram avaliados 6 indivíduos na classe de produção A, 3 indivíduos na classe $B$, enquanto que nas classes $C$ e $D$ foram avaliados 5 indivíduos em cada. 


\subsection{Amostragem e avaliações}

Em novembro de 2009 foram coletados solo e folhas para avaliar a fertilidade do solo, a colonização por fungos micorrízicos arbusculares (FMA) e o estado nutricional das árvores. Na área de influência de cada indivíduo foram coletadas quatro subamostras de solo para constituir uma amostra composta. As amostras de solo foram retiradas de cada quadrante dentro da área de projeção da copa, nas profundidades de $0-10 \mathrm{~cm}$ e $10-20 \mathrm{~cm}$, totalizando 38 amostras compostas. As amostras foram levadas ao laboratório para secagem ao ar, na sequência foram destorroadas e passadas em peneira com malha de $2 \mathrm{~mm}$ (Raij et al., 2001). Foram extraídos fósforo (P) e potássio (K) por Mehlich 1, com determinações por colorimetria (P) e fotometria de chama (K), conforme descrito em Donagema et al. (2011). Cálcio (Ca), magnésio (Mg) e alumínio (Al) trocável foram extraídos por solução de $\mathrm{KCl} 1 \mathrm{~mol} \mathrm{~L}^{-1} \mathrm{e}$ determinados por espectrofotometria de absorção atômica (Ca e $\mathrm{Mg}$ ) e por titulação (Al), conforme descrito em Raij et al. (2001). O pH foi determinado em água, enquanto que a acidez total foi determinada com solução de acetato de cálcio $1 \mathrm{~mol} \mathrm{~L}^{-1}$ (Raij et al., 2001). Foram calculados os valores da soma de bases (SB), capacidade de troca catiônica (CTC), saturação por bases (V\%) e saturação por alumínio (m\%).

Para análises referentes ao número total de esporos de FMA, as subamostras foram retiradas de cada quadrante dos mesmos indivíduos em que a avaliação da fertilidade do solo foi feita, dentro e fora da área de projeção da copa, na profundidade de $0-10 \mathrm{~cm}$, totalizando 38 amostras compostas. Os esporos foram extraídos de uma alíquota de $50 \mathrm{~g}$ de solo, pela técnica de peneiramento úmido (Gerdemann \& Nicolson, 1963), com quatro passagens pela malha da peneira. O material retido na última malha foi observado em microscópio estereoscópico, com aumento de 30 vezes, sendo realizada a contagem dos esporos.

$\mathrm{Na}$ amostragem para avaliação do estado nutricional foram coletadas as folhas metabolicamente mais ativas (totalmente expandidas e não senescentes) na fase do pico de floração. Para cada indivíduo, uma amostra composta foi constituída por folhas retiradas de ramos provenientes dos quatro quadrantes. As folhas nas duas extremidades dos ramos foram eliminadas, sendo selecionadas para a análise em laboratório apenas as folhas localizadas na porção central. Foram determinados os teores de N, P, K, Ca e Mg. O N foi extraído por meio da digestão sulfúrica, enquanto que os demais nutrientes, pela digestão nítrico-perclórica. A quantificação do $\mathrm{N}$ foi realizada pelo método semimicro Kjeldahl, a do P, por colorimetria, a do K, por fotometria de chama, enquanto que $\mathrm{Ca}$ e $\mathrm{Mg}$ foram determinados por espectrofotometria de absorção atômica (Malavolta et al., 1997).

\subsection{Análises estatísticas}

Por meio do software ASSISTAT (Silva \& Azevedo, 2002), foi realizada análise de variância (ANOVA) para modelo desbalanceado com um fator de tratamento, seguida pelo teste de Tukey para comparação de médias a 5\% de significância.

A análise discriminante foi utilizada para testar diferenças significativas entre classes de produção e determinar funções discriminantes que permitissem classificar novas árvores nas classes, evidenciando as variáveis responsáveis pela diferenciação. Utilizou-se o método Stepwise e, como critério de seleção, o Lambda de Wilks, que considera o valor da estatística F multivariada para o teste da diferença entre os centroides dos grupos. $\mathrm{O}$ método pressupõe que valores de F inferiores a 3,84 sejam eliminados da análise por possuírem baixo poder de discriminação. Foram testadas as correlações entre a variável discriminante e atributos do solo indicativos da acidez.

\section{RESULTADOS E DISCUSSÃO}

Na Tabela 1 são apresentados os valores do número de frutos total para as classes de produção, o diâmetro a altura do peito (DAP) e o número de esporos de fungos micorrízicos arbusculares encontrados no solo. Somente o número de frutos total diferiu entre as classes de produção.

$\mathrm{Na}$ Tabela 2 são apresentados os valores de atributos químicos do solo, os quais diferiram entre as classes de produção somente na profundidade de $0-20 \mathrm{~cm}$. Os teores de $\mathrm{Ca}$ e $\mathrm{Mg}$ no solo foram maiores na classe média de produção, enquanto que os teores de $\mathrm{Al}$, $\mathrm{H}+\mathrm{Al} \mathrm{e} \mathrm{m \%}$ foram maiores na classe alta de produção.

Os resultados dos teores de macronutrientes nas folhas da castanheira são apresentados na Tabela 3 , na qual observa-se que não houve diferença entre as classes de produção das árvores. 
Tabela 1. Médias, valores de F e coeficiente de variação $(\mathrm{CV})$ do número total de frutos $\left(\mathrm{NF}_{\text {total }}\right)$, do diâmetro a altura do peito (DAP) das árvores e do número de esporos de fungos micorrízicos arbusculares no solo coletado dentro da projeção (FMA P) e fora da projeção (FMA FP) da copa das castanheiras.

Table 1. Means, F values, and variation coefficient $(\mathrm{CV})$ of fruits total number $\left(\mathrm{NF}_{\text {total }}\right)$, of trees diameter at breast high (DAP), and of arbuscular mycorrhizal fungi spore number in the soil sampled both within (FMA P) and out (FMA FP) the canopy projection.

\begin{tabular}{|c|c|c|c|c|}
\hline \multirow{2}{*}{ Classes de produção } & $\mathbf{N F}_{\text {total }}$ & DAP & FMA P & FMA FP \\
\hline & frutos árvore ${ }^{-1}$ & -----cm------ & \multicolumn{2}{|c|}{------esporos $100 \mathrm{~g}^{-1} \mathrm{de}$ solo----- } \\
\hline Alta & $460,6 a$ & $107,7 \mathrm{a}$ & $583,4 \mathrm{a}$ & $714,4 \mathrm{a}$ \\
\hline Média & $321,2 b$ & $109,0 \mathrm{a}$ & $668,0 \mathrm{a}$ & $697,9 a$ \\
\hline Baixa & $168,6 c$ & $119,9 \mathrm{a}$ & $559,8 \mathrm{a}$ & $721,6 a$ \\
\hline Muito baixa & $38,6 \mathrm{~d}$ & $88,7 \mathrm{a}$ & $706,8 \mathrm{a}$ & $799,3 \mathrm{a}$ \\
\hline Valor F & $101,68^{\star *}$ & $2,78^{\text {ns }}$ & $0,72^{\text {ns }}$ & $0,40^{\text {ns }}$ \\
\hline $\mathrm{CV}(\%)$ & 17,2 & 15,9 & 39,0 & 25,4 \\
\hline
\end{tabular}

${ }^{n s}$ não significativo; ${ }^{* *}$ significativo a $1 \%$ de probabilidade; médias seguidas de mesma letra em cada coluna não diferem entre si a $5 \%$ de probabilidade pelo teste de Tukey.

Tabela 2. Médias, valores de F e coeficiente de variação $(\mathrm{CV})$ de atributos químicos em duas profundidades do solo (0-20 cm e 20-40 cm) para as classes de produção de frutos da castanheira-do-brasil.

Table 2. Means, F values, and variation coefficient (CV) of soil chemical attributes at two depths $(0-20$ and $20-40 \mathrm{~cm})$ to the production classes of Brazil nut tree fruits.

\begin{tabular}{|c|c|c|c|c|c|c|c|c|c|c|c|}
\hline \multirow{2}{*}{$\begin{array}{l}\text { Classes de } \\
\text { produção }\end{array}$} & \multirow[b]{2}{*}{$\mathrm{pH}_{\mathrm{H} 2 \mathrm{O}}$} & $\mathbf{P}$ & $\mathbf{K}$ & $\mathrm{Ca}$ & $\mathrm{Mg}$ & Al & $\mathrm{H}+\mathrm{Al}$ & SB & $\mathbf{T}$ & V & $\mathbf{m}$ \\
\hline & & $\begin{array}{c}\text { mg } \\
\mathrm{kg}^{-1}\end{array}$ & \multicolumn{7}{|c|}{ L-1 } & \multicolumn{2}{|c|}{--------\%------- } \\
\hline \multicolumn{12}{|c|}{ Profundidade 0-20 cm } \\
\hline Alta & $3,9 a$ & $6,5 a$ & $0,11 \mathrm{a}$ & $0,9 b$ & $0,8 b$ & $2,6 a$ & $10,4 a$ & $1,8 \mathrm{a}$ & $12,1 \mathrm{a}$ & $12,3 a$ & $60,3 a$ \\
\hline Média & $4,3 \mathrm{a}$ & $5,4 \mathrm{a}$ & $0,10 \mathrm{a}$ & $1,8 \mathrm{a}$ & $1,6 a$ & $1,5 b$ & $8,8 \mathrm{ab}$ & $3,5 \mathrm{a}$ & $12,4 \mathrm{a}$ & $29,8 \mathrm{a}$ & $30,8 b$ \\
\hline Baixa & $4,0 \mathrm{a}$ & $4,0 \mathrm{a}$ & $0,10 \mathrm{a}$ & $0,9 b$ & $0,8 b$ & $2,0 \mathrm{ab}$ & $8,7 \mathrm{ab}$ & $1,8 \mathrm{a}$ & $10,5 \mathrm{a}$ & $17,6 \mathrm{a}$ & $52,5 \mathrm{ab}$ \\
\hline Muito baixa & $4,4 \mathrm{a}$ & $6,3 a$ & $0,10 \mathrm{a}$ & $1,5 b$ & $1,3 \mathrm{ab}$ & $1,3 b$ & $7,4 \mathrm{~b}$ & $2,9 a$ & $10,6 a$ & $20,8 \mathrm{a}$ & $35,4 b$ \\
\hline Valor de F & $3,31^{\mathrm{ns}}$ & $1,99^{\text {ns }}$ & $0,55^{\mathrm{ns}}$ & $30,22^{\star *}$ & $6,43^{\star *}$ & $6,58^{* *}$ & $5,24^{*}$ & $3,22^{\text {ns }}$ & $1,89^{\mathrm{ns}}$ & $3,16^{\mathrm{ns}}$ & $4,71^{\star}$ \\
\hline CV (\%) & 7,5 & 31,5 & 19,2 & 54,7 & 39,6 & 29,5 & 13,4 & 46,5 & 15,0 & 59,4 & 34,0 \\
\hline \multicolumn{12}{|c|}{ Profundidade $20-40 \mathrm{~cm}$} \\
\hline Alta & $4,0 \mathrm{a}$ & $4,0 \mathrm{a}$ & $0,06 a$ & $0,6 \mathrm{a}$ & $0,6 \mathrm{a}$ & $2,3 a$ & $7,4 a$ & $1,3 \mathrm{a}$ & $8,7 a$ & $14,5 \mathrm{a}$ & $64,8 \mathrm{a}$ \\
\hline Média & $4,2 \mathrm{a}$ & $4,7 \mathrm{a}$ & $0,07 \mathrm{a}$ & $0,9 \mathrm{a}$ & $0,6 a$ & $1,7 \mathrm{a}$ & $6,3 \mathrm{a}$ & $1,6 \mathrm{a}$ & $7,8 \mathrm{a}$ & $20,1 \mathrm{a}$ & $51,7 \mathrm{a}$ \\
\hline Baixa & $4,1 \mathrm{a}$ & $3,0 \mathrm{a}$ & $0,07 \mathrm{a}$ & $0,4 \mathrm{a}$ & $0,4 \mathrm{a}$ & $1,8 \mathrm{a}$ & $6,0 \mathrm{a}$ & $1,0 \mathrm{a}$ & $7,0 \mathrm{a}$ & $15,2 \mathrm{a}$ & $63,6 a$ \\
\hline Muito baixa & $4,3 \mathrm{a}$ & $4,0 \mathrm{a}$ & $0,08 \mathrm{a}$ & $0,8 \mathrm{a}$ & $0,7 \mathrm{a}$ & $1,8 \mathrm{a}$ & $6,5 \mathrm{a}$ & $1,6 \mathrm{a}$ & $8,1 \mathrm{a}$ & $18,8 \mathrm{a}$ & $55,6 \mathrm{a}$ \\
\hline Valor de F & $2,11^{\mathrm{ns}}$ & $1,39^{\text {ns }}$ & $0,50^{\text {ns }}$ & $2,29^{\text {ns }}$ & $1,02^{\mathrm{ns}}$ & $1,54^{\mathrm{ns}}$ & $1,05^{\mathrm{ns}}$ & $1,40^{\text {ns }}$ & $1,06^{\mathrm{ns}}$ & $1,38^{\text {ns }}$ & $1,55^{\text {ns }}$ \\
\hline $\mathrm{CV}(\%)$ & 4,6 & 38,6 & 29,0 & 46,4 & 47,9 & 23,0 & 14,0 & 43,8 & 15,8 & 31,8 & 19,6 \\
\hline
\end{tabular}

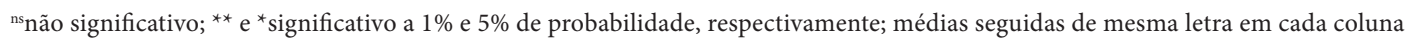
não diferem entre si a $5 \%$ de probabilidade pelo teste de Tukey.

Analisando-se os resultados dos atributos químicos do solo é possível constatar que os indicadores de acidez ( $\mathrm{Al} \mathrm{e} \mathrm{H}+\mathrm{Al}$ ) foram maiores no solo sob influência das árvores mais produtivas. Para confirmar essa relação, com os resultados de atributos químicos do solo e dos teores de nutrientes nas folhas da castanheira compôs-se uma matriz para a análise discriminante.

A variável $\mathrm{Al}_{(0-20)}$ foi selecionada como de real poder de discriminação (Tabela 4) e a determinação das funções discriminantes, importantes na análise da influência das variáveis na formação das classes de produção (Tabela 5), indicou ser possível determinar apenas uma função com poder de discriminação, que representou $100 \%$ da variância total.

A função classificatória classe de produção $=-3,598+1,851 \mathrm{Al}_{(0-20 \mathrm{~cm})}$ classificou corretamente em 52,9\% dos casos, podendo ser utilizada para classificar novas árvores com precisão razoável. Na Tabela 6 se observa o percentual de classificações corretas e incorretas efetuadas pelo modelo para as 
Tabela 3. Médias, valores de F e coeficiente de variação (CV) dos teores de macronutrientes determinados nas amostras de folhas da castanheira-do-brasil.

Table 3. Means, F values, and variation coefficient (CV) of macronutrient contents determined in leaf samples of Brazil nut trees.

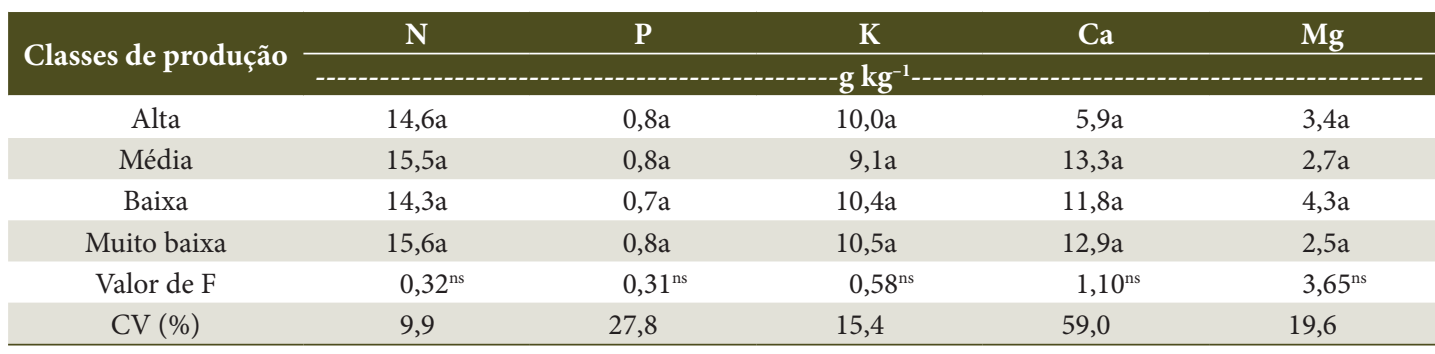

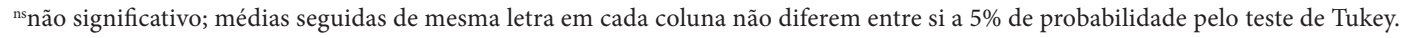

Tabela 4. Valor F e Lambda de Wilks para a variável selecionada.

Table 4. F value and Wilks' Lambda to the selected variable.

\begin{tabular}{cccc} 
Step & Variável & $\mathbf{F}$ & $\lambda^{*}$ \\
1 & $\mathrm{Al}_{0-20}$ & 4,67 & 0,44 \\
\hline
\end{tabular}

${ }^{*}$ significativo a $5 \%$ de probabilidade

Tabela 5. Seleção das funções discriminantes para a matriz de dados de árvores de castanha-do-brasil.

Table 5. Selection of discriminant functions to the matrix data of Brazil nut trees.

\begin{tabular}{ccccccccc} 
Função & Auto-valor & $\begin{array}{c}\% \text { da } \\
\text { variância }\end{array}$ & $\begin{array}{c}\text { \% variância } \\
\text { acumulada }\end{array}$ & $\begin{array}{c}\text { Correlação } \\
\text { canônica }\end{array}$ & $\lambda^{*}$ & $\chi^{2}$ & GL & Sig. \\
\hline 1 & 1,273 & $100 \%$ & $100 \%$ & 0,748 & 0,440 & 9,44 & 3 & 0,024 \\
\hline
\end{tabular}

${ }^{*}$ significativo a $5 \%$ de probabilidade

Tabela 6. Número de casos e porcentagem de classificação das árvores nas classes de produção.

Table 6. Number of cases and classification percentage of trees in the production classes.

\begin{tabular}{|c|c|c|c|c|c|}
\hline \multirow{2}{*}{$\begin{array}{l}\text { Classes de } \\
\text { produção }\end{array}$} & \multirow{2}{*}{$\begin{array}{l}\text { Número de } \\
\text { árvores }\end{array}$} & \multicolumn{4}{|c|}{ Classes de produção } \\
\hline & & Alta & Média & Baixa & Muito baixa \\
\hline Alta & 6 & $5(83 \%)$ & & & \\
\hline Média & 3 & $1(33 \%)$ & $2(67 \%)$ & $1(33 \%)$ & $1(33 \%)$ \\
\hline Baixa & 5 & $2(40 \%)$ & & & $2(40 \%)$ \\
\hline Muito baixa & 5 & & $1(20 \%)$ & & $4(80 \%)$ \\
\hline
\end{tabular}

árvores avaliadas. $\mathrm{O}$ maior percentual de acertos foi obtido para os grupos de produção alta e muito baixa, enquanto que nos grupos intermediários o percentual de acertos foi menor.

Foi aplicada a análise de variância univariada e foram identificadas diferenças significativas entre as classes de produção $(\mathrm{F}=4,992 ; \mathrm{P}=0,016)$ para o teor de $\mathrm{Al}_{(0-20 \mathrm{~cm})}$. A diferença entre médias realizadas pelo teste LSD indicou haver diferença estatística para o teor de $\mathrm{Al}_{(0-20 \mathrm{~cm})}$ no solo entre a classe $\mathrm{A}$ (árvores de alta produção) e as demais (Figura 1).

Foi constatada correlação negativa significativa entre $\mathrm{Al}_{(0-20)}$ e $\mathrm{pH}_{(0-20)}$ (Figura 2), $\mathrm{Al}$ e teores de Ca e
Mg (Figura 3), Al e V\% (Figura 4) e Al e SB (Figura 5). Por outro lado, foi observada correlação positiva entre $\mathrm{Al}_{(0-20)}$ e m\% (Figura 4) e $\mathrm{Al}_{(0-20)}$ e $\mathrm{H}+\mathrm{Al}$ (Figura 5).

Como os maiores coeficientes de determinação foram encontrados nas correlações com $\mathrm{pH}\left(\mathrm{R}^{2}=0,87\right)$ e $\mathrm{m} \%\left(\mathrm{R}^{2}=0,82\right)$, convém ressaltar que as árvores mais produtivas, além de estarem associadas a maior teor de alumínio na camada de $0-20 \mathrm{~cm}$ do solo, também estiveram associadas a menor valor de $\mathrm{pH}$ e menor quantidade de bases trocáveis na CTC. Cálcio (Ca) e magnésio $(\mathrm{Mg})$ foram as bases trocáveis mais relevantes, enquanto que o potássio (K), apesar de sua importância como macronutriente primário, apresentou fraca correlação $\left(\mathrm{R}^{2}=0,01\right)$ com o $\mathrm{Al}_{(0-20)}$. 


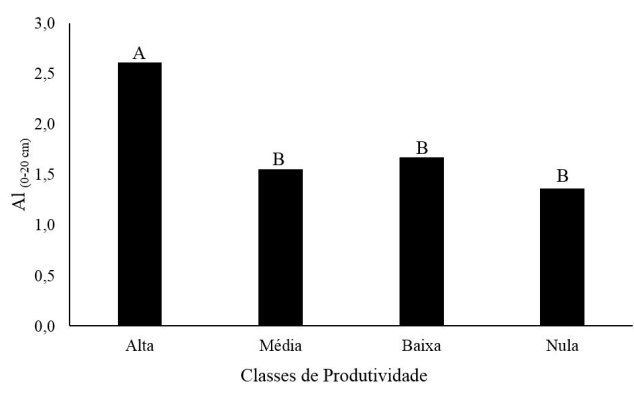

Figura 1. Teor médio de alumínio do solo na profundidade $0-20 \mathrm{~cm}$ em função das quatro classes de produção da castanheira-do-brasil. Médias seguidas de mesma letra não diferem entre si a 5\% de probabilidade pelo teste de Tukey.

Figure 1. Mean of aluminum content at soil depth of $0-20 \mathrm{~cm}$ as a function of four production classes of Brazil nut fruits. Means followed by same letter do not significantly $5 \%$ of probability by Tukey test.

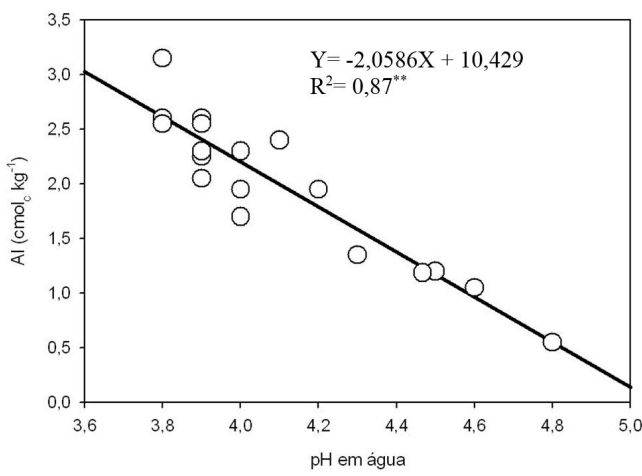

Figura 2. Teor de alumínio extraído do solo em função do $\mathrm{pH} .{ }^{* *}$ Significativo a $1 \%$ de probabilidade pelo teste F. Figure 2. Extracted soil aluminum content as a function of $\mathrm{pH}$. ${ }^{*}$ Significant at $1 \%$ of probability by $\mathrm{F}$ test.

A correlação negativa entre $\mathrm{pH}$ e $\mathrm{Al}$ no solo já é bem conhecida, pois com a redução do $\mathrm{pH}$ a valores inferiores a 5, o alumínio é solubilizado na solução do solo (Kochian et al., 2005). Além disso, convém mencionar que a acidificação está associada com as perdas de bases trocáveis, formação de matéria orgânica de baixa qualidade e em mobilização do Al potencialmente tóxico às plantas (Boruvka et al., 2005). A solubilidade $\mathrm{e}$ a concentração de Al na forma trocável são controladas pela acidez e os íons hidrogênio competem com elementos essenciais pelos mesmos sítios químicos (Ellis \& Mellor, 1995; Kochian et al., 2005), fazendo com que o $\mathrm{Al}$ seja considerado promotor da redução da fertilidade do solo (Amorim \& Batalha, 2007).
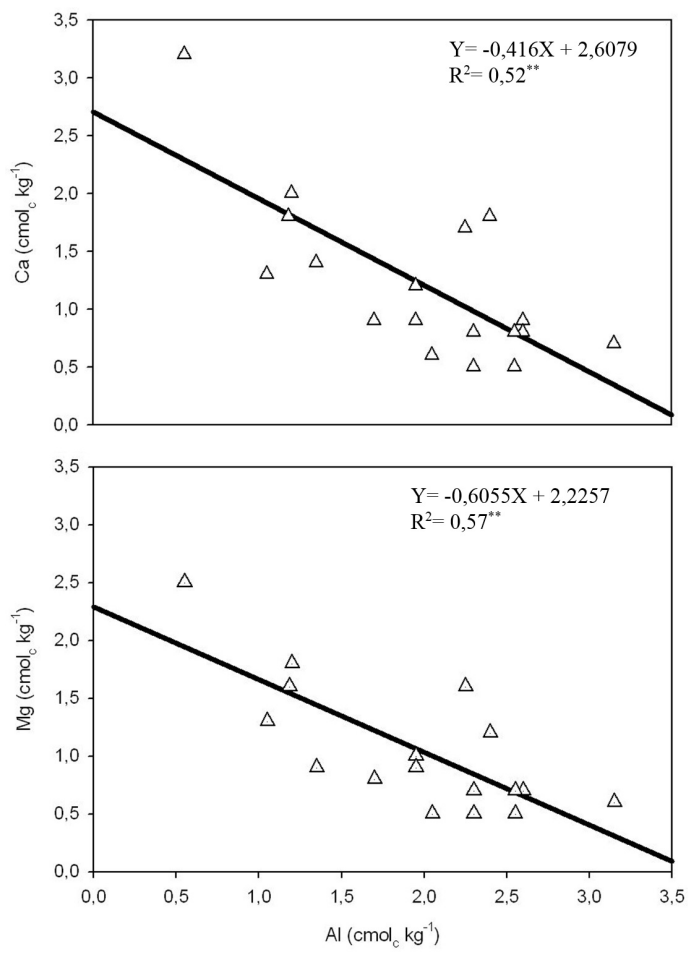

Figura 3. Relação entre aumento no teor de alumínio extraído do solo e bases trocáveis (Ca e $\mathrm{Mg}$ ). ** Significativo a $1 \%$ de probabilidade pelo teste F.

Figure 3. Relation between increases of soil extracted aluminum and exchangeable bases ( $\mathrm{Ca}$ and $\mathrm{Mg}$ ). ** Significant at $1 \%$ of probability by $\mathrm{F}$ test.

A vulnerabilidade inicial à acidificação é controlada naturalmente pelo material de origem associado aos fatores de formação dos solos (Boruvka et al., 2005). A acidificação pode ser causada por fatores de origem natural, tais como as reações geradoras de prótons e a absorção de cátions pelas plantas (Liu et al., 2010). Em se tratando da absorção, quando um cátion é absorvido pelas raízes das plantas há liberação de hidrogênio com a mesma equivalência, o que contribui para a acidificação (Duan et al., 2004). Contudo, atividades antropogênicas podem acelerar a acidificação dos solos, com destaque para a deposição ácida de enxofre e compostos nitrogenados (Richter, 1986; Kazda \& Zvacek, 1989; Boruvka et al., 2005) decorrentes das emissões de $\mathrm{SO}_{2}$ e $\mathrm{NO}_{\mathrm{x}}$ (Liu et al., 2010).

A acidificação de solos pela deposição ácida é mais relevante em áreas próximas às fontes de emissão (Boruvka et al., 2005). Como o local onde foi realizado este estudo está distante de áreas urbanas e industriais, a 

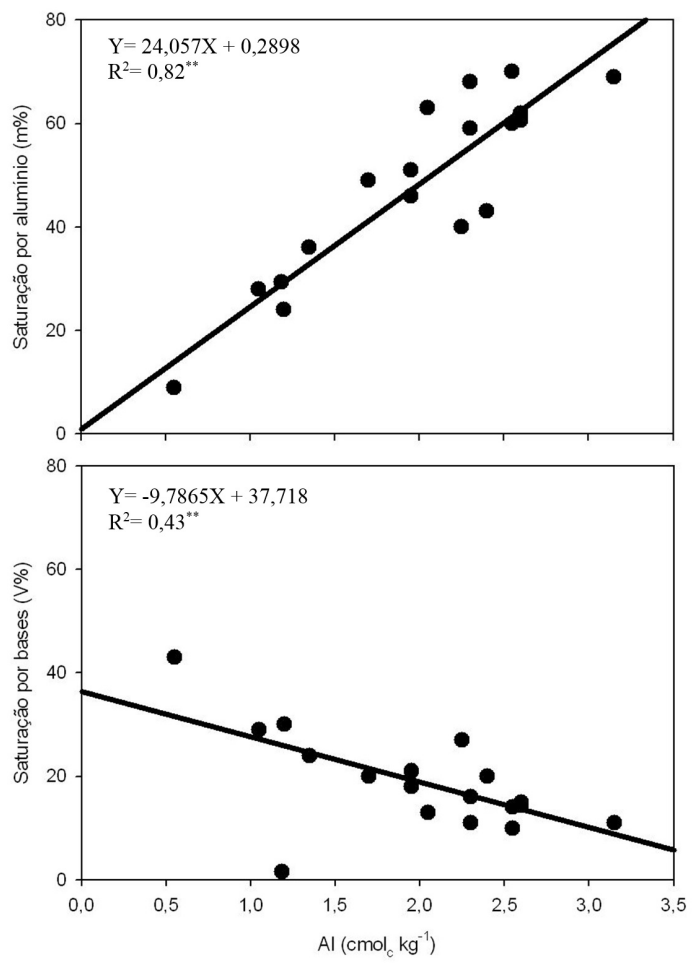

Figura 4. Relações entre aumento do alumínio trocável no solo, a saturação por alumínio $(\mathrm{m} \%)$ e a saturação por bases $(\mathrm{V} \%)$. ${ }^{*}$ Significativo a $1 \%$ de probabilidade pelo teste $\mathrm{F}$.

Figure 4. Relation between the increase of soil extracted aluminum and both saturation by aluminium ( $\mathrm{m} \%)$ and saturation by bases (V\%). ${ }^{* *}$ Significant at $1 \%$ of probability by $\mathrm{F}$ test.

explicação para a redução no $\mathrm{pH}$ está mais relacionada à remoção de bases em função da absorção de cátions pelas castanheiras e plantas do sub-bosque. Liu et al. (2010) observaram redução mais acentuada no pH de solos florestais em áreas onde a sucessão da vegetação apresentava estágio mais avançado. Os autores explicam o observado a partir do maior acúmulo de bases na biomassa.

A avaliação do estado nutricional confirmou a elevada absorção de bases do solo pela castanheira, pois as árvores apresentaram nas folhas teores de cálcio em quantidade semelhante à de espécies florestais cujas exigências são reconhecidamente elevadas (Tabela 3). Com exceção das árvores na classe alta de produção, cujo teor foliar de Ca foi de $5,8 \mathrm{~g} \mathrm{~kg}^{-1}$, o teor foliar do nutriente encontrado nas plantas ficou entre $11,8 \mathrm{~g} \mathrm{e} 13,3 \mathrm{~g} \mathrm{~kg}^{-1}$. Esses valores foram próximos ao limite superior da faixa de suficiência definida
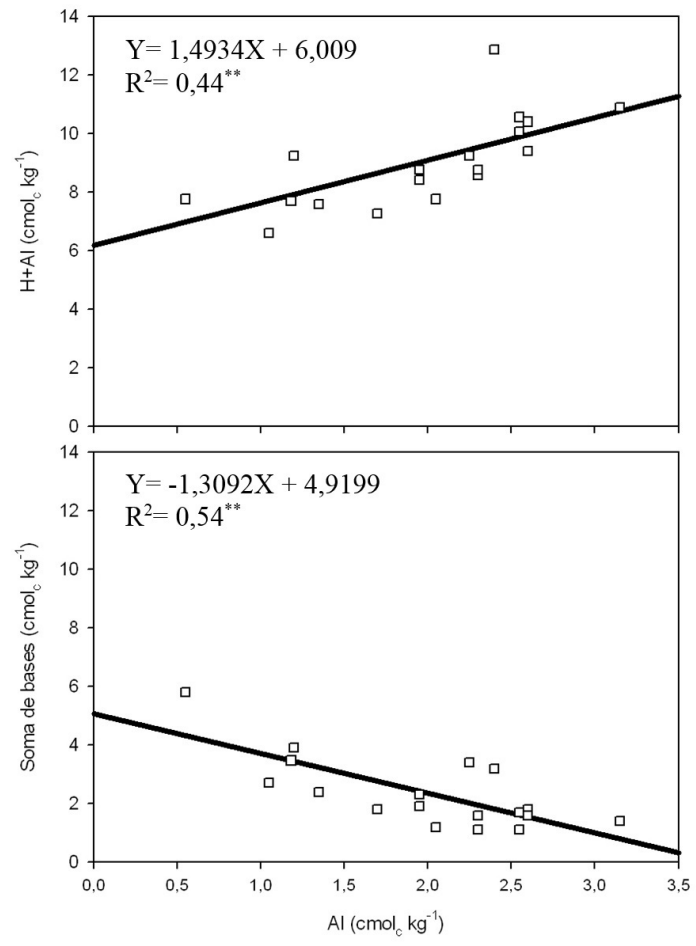

Figura 5. Acidez potencial $(\mathrm{H}+\mathrm{Al})$ e soma de bases (SB) em função do $\mathrm{pH}$ do solo. ${ }^{*}$ Significativo a $1 \%$ de probabilidade pelo teste $\mathrm{F}$.

Figure 5. Potential acidity $(\mathrm{H}+\mathrm{Al})$ and sum of bases (SB) as a function of soil $\mathrm{pH}$. ${ }^{*}$ Significant at $1 \%$ of probability by F test.

para o gênero eucalipto (8-12 $\left.\mathrm{g} \mathrm{kg}^{-1}\right)$ que, dentre as essências florestais, é considerado exigente em cálcio (Malavolta et al., 1997). Camargos (1999), analisando o teor de nutrientes em mudas de castanheira-do-brasil de 90 dias, encontrou valores de 15,4 g e 15,9 $\mathrm{g} \mathrm{kg}^{-1}$ de Ca para folhas novas e velhas, respectivamente. Schroth et al. (2015) encontraram 9,5 $\mathrm{g} \mathrm{kg}^{-1}$ de cálcio em folhas maduras de castanheiras. Vale destacar que para outras essências florestais consideradas menos exigentes em cálcio a faixa de suficiência apresenta valores inferiores a $8 \mathrm{~g} \mathrm{~kg}^{-1}$.

As árvores mais produtivas avaliadas neste estudo apresentaram menores teores foliares de Ca em relação às menos produtivas, possivelmente em função do efeito de diluição. Entretanto, tronco, caules, ramos, folhas e frutos constituem importantes compartimentos de estoque do nutriente (Schroth et al., 2015). O Ca é um importante elemento para a estrutura da parede celular e para vários processos fisiológicos em espécies arbóreas. 
Uma vez que o nutriente é estocado em uma parte da planta, ele não pode ser translocado para outras partes (Brady, 1969). Devido à grande quantidade de Ca no tecido madeireiro, Perakis et al. (2006) alertam para o fato de que a retirada sucessiva da madeira pode levar à deficiência de cálcio em florestas.

É certo que em castanhais onde é feita a exploração não madeireira a ciclagem de nutrientes contribui com o retorno ao solo dos nutrientes acumulados na biomassa das árvores. Mas, segundo Ticktin (2004), a extensão com a qual a ciclagem é alterada depende da intensidade do extrativismo e da parte da planta que é coletada. Os nutrientes que estiverem contidos nos frutos e sementes coletadas serão exportados da área e, no caso da castanha-do-brasil, alguns estudos mostram que as amêndoas possuem elevada quantidade de cálcio, de magnésio e de potássio (Gonçalves et al., 2002; Silva et al., 2010).

Mesmo com o importante papel da ciclagem de nutrientes em solos florestais, estratégias de manejo devem ser estabelecidas visando reposição de bases ao solo devido à coleta extrativista da castanha-do-brasil. Em se tratando de plantios estabelecidos, os cuidados com a reposição devem ser redobrados, partindo-se do princípio de que a intensidade de exploração é maior. A poda de castanheiras em SAF é recomendada por Schroth et al. (2015), que destacam, dentre os benefícios dessa prática, o favorecimento da ciclagem de cálcio e magnésio.

Não foi possível estabelecer relações entre disponibilidade de fósforo no solo, número de esporos de FMA, teor foliar de $\mathrm{P}$ e o número de frutos produzidos. Seguindo os critérios de interpretação da análise de solo para P extraído pelo método Mehlich 1 (Sousa \& Lobato, 2004), as concentrações do nutriente no solo sob os castanhais foram muito baixas $\left(3,9 \mathrm{mg} \mathrm{kg}^{-1}\right.$ na camada de $0-20 \mathrm{~cm} \mathrm{e} 1,2 \mathrm{mg} \mathrm{kg}^{-1}$ na camada de $20-40 \mathrm{~cm}$ ) (Tabela 2). Isso refletiu no teor foliar do nutriente $\left(0,8 \mathrm{~g} \mathrm{~kg}^{-1}\right)$, que ficou abaixo da faixa de suficiência definida para algumas essências florestais que têm sido mais estudadas (Malavolta et al., 1997) (Tabela 3).

Considerando o baixo teor de fósforo no solo sob influência das castanheiras e o baixo teor do nutriente no tecido vegetal das árvores, constata-se que não houve efeito da presença de FMA para favorecer a absorção de P. Contudo é interessante verificar que o número de esporos de FMA das amostras coletadas dentro da projeção da copa das castanheiras foi menor (638,2 esporos $100 \mathrm{~g}^{-1}$ de solo) que o número de esporos encontrado nas amostras coletadas fora da projeção da copa $\left(742,9\right.$ esporos $100 \mathrm{~g}^{-1}$ de solo $)(\mathrm{P}=0,03)$.

\section{CONCLUSÕES}

A castanheira-do-brasil mostrou-se exigente em cálcio, evidenciando a importância de técnicas de manejo visando a reposição do nutriente, como, por exemplo, pela deposição de resíduos de poda e das cascas das amêndoas e dos ouriços da castanheira sobre o solo.

As árvores mais produtivas removem mais bases trocáveis, contribuindo para a acidificação e empobrecimento do solo, exigindo cuidados no manejo tanto de castanhais naturais, onde se faz o extrativismo, como em castanhais plantados.

Não foi identificada relação entre o número de esporos de fungos micorrízicos arbusculares e a nutrição mineral das árvores em relação ao fósforo.

\section{STATUS DA SUBMISSÃO}

Recebido: 12 out., 2016

Aceito: 3 nov., 2016

\section{AUTOR(ES) PARA CORRESPONDÊNCIA}

\section{Mirian Gomes Costa}

Departamento de Ciências do Solo, Universidade Federal do Ceará - UFC, Avenida Mister Hull, 2977, Bloco 807, CEP 60165-180, Fortaleza, CE, Brasil

e-mail: mirian.costa@ufc.br

\section{REFERENNCIAS}

Amorim PK, Batalha MA. Soil vegetation relationships in hyperseasonal cerrado, seasonal cerrado, and wet grassland in Emas National Park (Central Brazil). Acta Oecologica 2007; 32(3): 319-327. http://dx.doi.org/10.1016/j. actao.2007.06.003.

Bernatchez F, Jeannotte R, Begg CBM, Hamel C, Whalen JK. Soil Fertility and arbuscular mycorrhizal fungi related to trees growing on smallholder farms in Senegal. Journal of Arid Environments 2008; 72(7): 1247-1256. http://dx.doi. org/10.1016/j.jaridenv.2007.12.014. 
Boruvka L, Mladkova L, Drabek O. Factors controlling spatial distribution of soil acidification and $\mathrm{Al}$ forms in Forest soils. Journal of Inorganic Biochemistry 2005; 99(9): 1796-1806. PMid:16095712. http://dx.doi.org/10.1016/j. jinorgbio.2005.06.028.

Brady SL. The effect of mineral nutrition on the anatomy of Larix occidentalis (Nutt.) seedlings [tese]. State College: Pennsylvania State University; 1969.

Brasil. Departamento Nacional da produção Mineral. Projeto RADAMBRASIL. Folha NA 20 Boa Vista e parte das folhas NA 21, Tumucumaque, N. 20 Roraima e NB 21; geologia, geomorfologia, pedologia, vegetação e uso potencial da terra. Rio de Janeiro, 1975. 428 p.

Camargos SL. Diagnose de deficiência, teor e acúmulo de nutrientes em castanheira-do-brasil [tese]. Piracicaba: Centro de Energia Nuclear na Agricultura; 1999.

Donagema GK, Campos DVB, Calderano SB, Teixeira WG, Viana JHM. Manual de métodos de análise de solos. Rio de Janeiro: Embrapa Solos; 2011.

Duan L, Huang Y, Hao J, Xie S, Hou M. Vegetation uptake of nitrogen and base cations in China and its role in soil acidification. The Science of the Total Environment 2004; 330(1-3): 187-198. PMid:15325168. http://dx.doi. org/10.1016/j.scitotenv.2004.03.035.

Ellis S, Mellor A. Soils and environment. New York: Routledge; 1995.

Fundação do Meio Ambiente Ciência e Tecnologia do Estado de Roraima - FEMACT. O Brasil do hemisfério norte: diagnóstico científico e tecnológico para o desenvolvimento. Boa Vista: Ambtec; 1993.

Gerdemann JW, Nicolson TH. Spores of mycorrhizal Endogone species extracted from soil by wet sieving and decanting. Transactions of the British Mycological Society 1963; 46(2): 235-244. http://dx.doi.org/10.1016/ S0007-1536(63)80079-0.

Gonçalves JFC, Fernandes AV, Oliveira AFM, Rodrigues LF, Marenco RA. Primary metabolism components of seeds from Brazilian Amazontree species. Brazilian Journal of Plant Physiology 2002; 14(2): 139-142. http://dx.doi. org/10.1590/S1677-04202002000200009.

Huguenin GVB, Oliveira GMM, Moreira ASB, Saint'Pierre TD, Gonçalves RA, Pinheiro-Mulder AR et al. Improvement of antioxidante status after Brazil nut intake in hypertensive and dylidemic subjects. Nutrition Journal 2015; 14(54): 1-10. PMid:25554072.

Kainer KA, Wadt LHO, Staudhammer CL. Explaining variation in Brazil nut fruit production. Forest Ecology and Management 2007; 250(3): 244-255. http://dx.doi. org/10.1016/j.foreco.2007.05.024.

Kazda M, Zvacek L. Aluminium and manganese and their relation to calcium in soil solution and needles in three Norvay spruce (Picea abies, L. Krast.) stands of Upper
Austria. Plant and Soil 1989; 114(2): 257-267. http:// dx.doi.org/10.1007/BF02220806.

Kluczkovski AM, Martins M, Mundim SM, Simões RH, Nascimento KS, Marinho HA et al. Properties of Brazil nuts: a review. African Journal of Biotechnology 2015; 14(8): 642-648. http://dx.doi.org/10.5897/AJB2014.14184.

Kochian LV, Piñeros MA, Howkenga OA. The physiology, genetics and molecular biology of plant aluminum resistance and toxicity. Plant and Soil 2005; 274(1-2): 175-195. http://dx.doi.org/10.1007/s11104-004-1158-7.

Lathwell DJ, Grove TL. Soil-plant relationships in the tropics. Annual Review of Ecology and Systematics 1986; 17(1): 1-16. http://dx.doi.org/10.1146/annurev. es.17.110186.000245.

Liu KH, Fang YT, Yu FM, Liu Q, Li FR, Peng SL. Soil acidification in response to acid deposition in three subtropical forests of subtropical China. Pedosphere 2010; 20(3): 399-408. http://dx.doi.org/10.1016/S10020160(10)60029-X.

Malavolta E, Vitti GC, Oliveira SA. Avaliação do estado nutricional das plantas: princípios e aplicações. 2. ed. Piracicaba: POTAFÓS; 1997.

Olsson PA, Thingstrup I, Jakobsen I, Baath E. Estimation of the biomass of arbuscular mycorrhizal fungi in a linseed field. Soil Biology \& Biochemistry 1999; 31(13): 1879-1887. http://dx.doi.org/10.1016/S0038-0717(99)00119-4.

Perakis SS, Maguire DA, Bullen TD, Cromack K, Waring $\mathrm{RH}$, Boyle JR. Coupled nitrogen and calcium cycles in forests of the Oregon Coast Range. Ecosystems (New York, N.Y.) 2006; 9(1): 63-74. http://dx.doi.org/10.1007/ s10021-004-0039-5.

Raij B, Andrade JC, Cantarella H, Quaggio JA. Análise química para avaliação da fertilidade de solos tropicais. Campinas: Instituto Agronômico; 2001.

Rayman MP. The argument for increasing selenium intake. Proceedings of the nutrition Society 2002; 61(2): 203-215. http://dx.doi.org/10.1079/PNS2002153.

Richter DD. Sources of acidity in some forested Udults. Soil Science Society of America Journal 1986; 50(6): 15841589. http://dx.doi.org/10.2136/sssaj1986.0361599500 5000060040x.

Schroth G, Mota MSS, Elias MEA. Growth and nutrient accumulation of Brazil nut trees (Bertholletia excelsa) in agroforestry at different fertilizer levels. Journal of Forest Research 2015; 26(2): 347-353. http://dx.doi.org/10.1007/ s11676-015-0037-9.

Silva FAS, Azevedo CAV. Versão do programa computacional Assistat para o sistema operacional Windows. Revista Brasileira de Produtos Agroindustriais 2002; 4(1): 71-78. http://dx.doi.org/10.15871/1517-8595/rbpa.v4n1p71-78.

Silva RF, Ascheri JLR, Souza JML. Influência do processo de beneficiamento na qualidade de amêndoas de castanha- 
do-brasil. Ciência e Agrotecnologia 2010; 34(2): 445-450. http://dx.doi.org/10.1590/S1413-70542010000200025.

Sousa DMG, Lobato E. Cerrado: correção do solo e adubação. 2. ed. Brasília: Embrapa Informação Tecnológica; 2004.

Ticktin T. The ecological implications of harvesting non-timber Forest products. Journal of Applied Ecology 2004; 41(1): 11-21. http://dx.doi.org/10.1111/j.13652664.2004.00859.x.

Tonini H, Costa P, Kaminski PE. Estrutura e produção de duas populações nativas de castanheira-do-Brasil (Bertholletia excelsa O. Berg) em Roraima. Floresta 2008; 38(3): 445-457. http://dx.doi.org/10.5380/ rf.v38i3.12410.
Viana VM, Mello RA, Morais LM, Mendes NT. Ecologia e manejo de populações de castanha-do-pará em reservas extrativistas Xapurí, Estado do Acre. In: Gascon C, Montinho P, editores. Floresta Amazônica: dinâmica, regeneração e manejo. Manaus: Instituto Nacional de Pesquisa da Amazônia; 1998.

Wunder S, Angelsen A, Belcher B. Forests, livelihoods, and conservation: broadening the empirical base. World Development 2014; 64: S1-S11. http://dx.doi.org/10.1016/j. worlddev.2014.03.007.

Zuidema PA. Ecology and management of the Brazil nut tree (Bertholletia excelsa): PROMAB - Programa Manejo de Bosques de la Amazônia Boliviana. Riberalta: PROMAB; 2003. Scientific Series 6. 